\title{
Generalized Mom-structures and ideal triangulations of 3-manifolds with nonspherical boundary
}

\author{
EKATERINA PERVOVA
}

\begin{abstract}
The so-called Mom-structures on hyperbolic cusped 3-manifolds without boundary were introduced by Gabai, Meyerhoff, and Milley, and used by them to identify the smallest closed hyperbolic manifold. In this work we extend the notion of a Mom-structure to include the case of 3-manifolds with nonempty boundary that does not have spherical components. We then describe a certain relation between such generalized Mom-structures, called protoMom-structures, internal on a fixed 3 -manifold $N$, and ideal triangulations of $N$; in addition, in the case of nonclosed hyperbolic manifolds without annular cusps, we describe how an internal geometric protoMom-structure can be constructed starting from the Epstein-Penner or Kojima decomposition. Finally, we exhibit a set of combinatorial moves that relate any two internal protoMom-structures on a fixed $N$ to each other.
\end{abstract}

57M20, 57N10; 57M15, 57M50

\section{Introduction}

This paper is devoted to an extension of the notion of Mom-structure, that is defined for manifolds with toric boundary, to the case of manifolds with arbitrary nonspherical nonempty boundary. Mom-structures were introduced by Gabai, Meyerhoff and Milley [6; 4] in the context of hyperbolic 3-manifolds and served as a tool for them to identify the smallest closed hyperbolic manifold [4; 12]. Certain components of a similar notion for compact hyperbolic manifolds with nonempty totally geodesic boundary were considered by Kojima and Miyamoto [10] and Deblois and Shalen [1], also as an instrument for studying volume.

A Mom-structure [6] (see Section 1 below for the precise definition) is a triple of form $(M, T, \Delta)$, where $M$ is a $3-$ manifold with toric boundary, $T$ is a selected torus in $\partial M$, and $\Delta$ is a particular type of handle decomposition of $M$ that includes a collar of $T$ serving as the "base" for the subsequent gluings of 1-and 2-handles. The crucial point is then to consider a fixed hyperbolic manifold $N$ and the set of Mom-structures internal on $N$ (roughly speaking, the structure is internal on $N$ if $N$ can be reconstructed from the structure via some Dehn fillings). The relation with 
the hyperbolic volume, as suggested in [6] and then realized in [4], is, very generally, the following one. Given a complete finite-volume cusped hyperbolic manifold $N$, consider a horotorus neighbourhood of some cusp. After expanding it in the normal direction, it will eventually encounter itself, which gives rise to a 1-handle, while further expansions create other 1-and 2-handles. For $N$ of low enough volume, this process allows one to construct an internal Mom-structure on $N$ with at most three 2-handles (and in fact other properties), which is a crucial result of [4].

Considering examples of hyperbolic 3-manifolds with low Matveev complexity (available in the census of Frigerio, Martelli and Petronio [3]) that admit an ideal triangulation into hyperbolic tetrahedra suggests that something similar may occur for other types of hyperbolic 3-manifolds, particularly for "mixed" ones with both toric cusps and totally geodesic boundary. This fact served as a motivation for us to consider a slightly generalized notion of a Mom-structure, that we call a protoMom-structure, and to study its relations with ideal triangulations; we immediately note that we do this from a combinatorial point of view rather than from a geometric one.

The generalization itself is entirely straightforward; essentially, it consists in replacing $T$ with an arbitrary nonempty surface $\Sigma$ without spherical components. Our first, and perhaps not unexpected, result is that any such generalized Mom-structure internal on a fixed 3-manifold $N$ with boundary $\Sigma$ can actually be obtained by thickening some of the arcs and faces of a suitably chosen ideal triangulation of $N$ (Theorem 2.4). We emphasize that our proof of this fact has a constructive nature: how the desired triangulation can be constructed via a sequence of a small number of specific moves.

It is the construction just mentioned, rather than just the existence itself of the triangulation, that enables us to address the main point of the paper, namely, the determination of a set of combinatorial moves that relate any two protoMom-structures (internal on the same manifold) to each other. The definition of the moves given in Section 4 is rather natural in view of Theorem 2.4 and of the easy fact that an ideal triangulation naturally gives rise to a variety of internal protoMom-structures (Section 2.1). Indeed, protoMom-structures arise from a triangulation by discarding some of its faces and by thickening the remaining ones, and by possibly cancelling some 1 -handles (that are thickenings of the edges) of valence 1 with the respective 2-handles. The way in which one can change the choice of which faces will be discarded naturally translates into certain M-moves, while the collapses of 1-handles translate into so-called $C$-moves. It is then the content of Theorem 4.6 that these moves are indeed sufficient to relate between them any two protoMom-structures internal on a fixed manifold.

The structure of the paper is as follows. Section 1 contains the necessary definitions, while Section 2 describes the relations between ideal triangulations and protoMomstructures. Section 3 is devoted to establishing some preliminary results needed to 
prove Theorem 4.6, and Section 4 contains the definitions of the moves together with the proof of the main theorem. Throughout the paper we will employ the piecewise linear viewpoint, which is equivalent to the smooth one in dimensions 2 and 3.

Acknowledgments This work was carried out while the author was holding a Junior Visiting position at the Centro De Giorgi of the Scuola Normale Superiore of Pisa, whose staff the author would like to thank for the excellent working conditions. Mathematical discussions with other postdocs at the Centro, particularly with Pablo Alvarez-Caudevilla, Antoine Lemenant and Laura Spinolo, were of indirect, but notable, benefit for the completion of this paper. The author would also like to thank the members of the research group in geometry at the Department of Mathematics of the University of Pisa for their interest, in particular Roberto Frigerio for the useful suggestions provided and Carlo Petronio for the very detailed comments on the exposition style of the first draft of this paper. Finally, the author wishes to thank the anonymous referee for the useful remarks on the first submitted version.

This work was partially supported by RFFI (project 10-01-96035) and by the Program "Mathematical problems of algebra, topology, function approximation theory, and applications" carried out jointly by the Institute for Mathematics and Mechanics UrO RAN and the Institute for Mathematics SO RAN.

\section{Preliminaries and main definitions}

In this section we recall some known definitions and facts that will be used in the rest of the paper, and we describe the main object of our study, which is a generalization of the key definition of [6] recalled below.

Mom-structures Given a handle decomposition of some cobordism, we will call valence of a 1-handle the number of 2-handles incident to it (with multiplicity), and valence of a 2-handle the number of 1-handles to which it is incident (with multiplicity). The original definitions due to Gabai, Meyerhoff and Milley are as follows.

Definition 1.1 [6, Definition 0.4] A Mom- $n$ structure is a triple $(M, T, \Delta)$ where

- $\quad M$ is a compact connected 3-manifold such that $\partial M$ is a union of tori;

- $T$ is a preferred boundary component of $M$;

- $\Delta$ is a decomposition of $M$ obtained as follows:

- take $T \times[0,1] \subset M$ such that $T \times\{0\}=T$;

- attach $n$ 1-handles to $T \times\{1\}$;

- add $n$ 2-handles of valence 3 in such a way that each 1 -handle has valence at least 2 . 
Definition 1.2 Let $M$ be a compact connected 3-manifold, and let $S \subset \partial M$ be a compact surface, which can be disconnected or empty. A general-based handle structure $\Delta$ on $(M, S)$ is a decomposition of $M$ obtained in the following way:

- take $S \times[0,1] \subset M$ such that $S \times\{0\}=S$;

- add several 0-handles;

- attach finitely many $1-$ and 2 -handles to $S \times\{1\}$ and to the 0 -handles;

- if needed, fill in some spherical boundary components with 3-handles.

Thus, if $(M, T, \Delta)$ is a Mom-structure then $\Delta$ is a (particular type of a) general-based handle decomposition on $(M, T)$.

Given a general-based handle decomposition $\Delta$ on $(M, S)$, we will call

- islands the connected components of the intersection of the 1-handles of $\Delta$ with $(S \times\{1\}) \cup\{0$-handles $\}$;

- bridges the connected components of the intersection of the 2-handles of $\Delta$ with $(S \times\{1\}) \cup\{0$-handles $\}$;

- lakes the connected components of the complement in $(S \times\{1\}) \cup\{$ boundaries of 0 -handles $\}$ of the union of all islands and bridges.

Definition 1.3 Let $\Delta$ be a general-based handle structure on $(M, S)$. Then $\Delta$ is full if all the lakes are discs.

ProtoMom-structures and weak protoMom-structures The main object of our study throughout the paper will be the following specific type of a general-based handle decomposition, that is obtained by a (rather straightforward) generalization of the notion of a Mom-structure.

Definition 1.4 Let $M$ be a compact connected orientable 3-manifold such that $\partial M$ can be written as the union of a nonempty surface $\Sigma$ without spherical components, and some tori. Let $\Delta$ be a general-based handle decomposition on $(M, \Sigma)$ such that $\Delta$ does not contain 0 - or 3-handles, each 1-handle has valence at least 2, and each 2-handle has valence precisely 3 . Then the triple $(M, \Sigma, \Delta)$ is called a protoMom-structure.

Remark 1.5 Let $\Delta$ be a protoMom-structure on $M$ with $\partial M$ the union of a surface $\Sigma$ of the above type and some tori. Suppose that $\Delta$ contains $n 2$-handles; then $\Delta$ contains $n-g+11$-handles, where $g$ is the genus of $\Sigma$ (or, if $\Sigma$ is disconnected, the sum of the genera of its connected components). 
Remark 1.6 In the case where $\Sigma$ is a torus, a protoMom structure is simply a Mom structure in the sense of [6] (see also [4]).

Definition 1.7 Let $\Delta$ be a general-based handle decomposition on $(M, \Sigma)$ with $M$ and $\Sigma$ as in Definition 1.4. The triple $(M, \Sigma, \Delta)$ is called a weak protoMom-structure if $\Delta$ has no 0 - or 3-handles and all its 2 -handles have valence 3 (whereas no restriction is placed on the valences of 1 -handles).

Remark 1.8 Our definition of weakness differs from that given for Mom-structures in [6], where a weak Mom-structure is one where 2-handles are allowed to have valence 2 (for us it is always 3), while 1-handles are still required to have valence at least 2 (whereas we drop this assumption in the definition of weakness).

Lateral tori and internal protoMom-structures Given a (weak) protoMom-structure $(M, \Sigma, \Delta)$, we call any toric boundary component of $M$ that is not contained in $\Sigma$ a lateral torus of the given protoMom-structure. Clearly, if $\Sigma$ does not have toric boundary components, then any torus in $\partial M$ is lateral.

Definition 1.9 Let $N$ be a 3-manifold with nonempty boundary, and let $(M, \Sigma, \Delta)$ be a protoMom structure on a submanifold $M \subset N$. The triple $(M, \Sigma, \Delta)$ is called an internal protoMom structure on $N$ if $N \backslash M$ consists of a collar of $\partial N$ and possibly some solid tori.

The meaning of this definition is simply that $N$ can be obtained from $M$ by Dehn-filling some of the lateral tori of $M$.

Note that in what follows our main focus will mostly be on full weak protoMomstructures internal on a certain fixed 3-manifold; we conclude this section by describing three other notions that will provide us with the tools necessary to obtain our results, namely ideal triangulations, special spines and their singular graphs, and the natural duality between the former two classes of objects.

Ideal triangulations $\mathrm{By}$ an ideal triangulation of a compact 3-manifold $N$ with boundary we mean a realization of $N$ as the result of first gluing a finite number of tetrahedra along a complete system of simplicial pairings of their lateral faces, and then removing small open neighbourhoods of all the vertices (such neighbourhoods must be small enough so that their closures be disjoint). Note that $N$ is thus decomposed into truncated tetrahedra (see Figure 2(left)), not into actual ones. Observe also that we allow multiple and self-adjacencies of the tetrahedra. 
Special spines A compact 2-dimensional polyhedron $P$ is called special if the following two conditions hold. First, the link of each point should be homeomorphic to one of the following 1-dimensional polyhedra:

(a) a circle;

(b) a circle with a diameter;

(c) a circle with three radii.

(See Figure 1 where the corresponding three possible types of regular neighbourhoods of a point of $P$ are shown). Second, the components of the set of points with link of
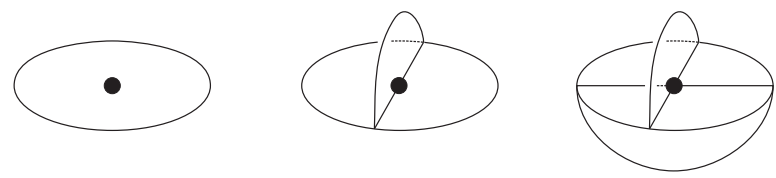

Figure 1. Neighbourhoods of points in a special polyhedron

type (a) are open discs, while the components of the set of points with link of type (b) are open segments.

The components just described are called faces and edges, respectively, and the points with link of type (c) are called vertices. The points with link of type (b) or (c) are called singular, and the set of all singular points of $P$ is denoted by $S(P)$, which is a four-valent graph (without circular components) that we will refer to as the singular graph of $P$.

Let now $N$ be a 3-manifold with nonempty boundary, and let $P$ be a special polyhedron embedded in Int $N$. We say that $P$ is a special spine of $N$ if $N \backslash P$ is an open collar of $\partial N$.

Duality The final notion that we need is the well-known duality between ideal triangulations of 3-manifolds with boundary and their special spines, summarized in the next statement.

Proposition 1.10 Let $N$ be a compact 3-manifold with nonempty boundary. Then the set of ideal triangulations of $N$ corresponds bijectively to the set of special spines of $N$ via the correspondence shown in Figure 2.

Given an ideal triangulation $\tau$ of $N$, we will denote the special spine dual to $\tau$ by $P_{\tau}$. 

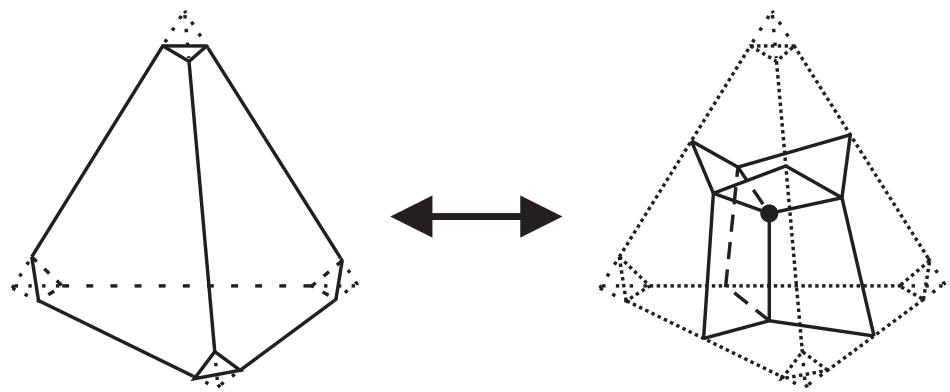

Figure 2. Duality between ideal triangulations and special polyhedra

\section{Ideal triangulations and internal protoMom-structures}

In this section we describe a procedure that, given an ideal triangulation of a manifold $N$ with nonempty boundary (without spherical components), allows us to obtain an internal weak protoMom-structure on $N$. We then give a slightly more general notion of a so-called triangulation-induced protoMom-structure (that we usually call $\tau$-induced, where $\tau$ stands for a specific triangulation), and we show that all full weak protoMomstructures internal on a given $N$ are actually induced by some ideal triangulation of $N$. The proof of this fact is constructive, and will be used in Section 4 to study combinatorial moves relating different protoMom-structures internal on the same fixed manifold $N$.

\subsection{Triangulation-induced protoMom-structures}

Fix a compact connected orientable 3-manifold $N$ such that $\partial N=\Sigma$ is a nonempty surface without spherical components. Let $\tau$ be an arbitrary ideal triangulation of $N$, seen as a decomposition of $N$ into truncated tetrahedra. Then $\tau$ gives rise to a number of internal protoMom-structures on $N$, which are constructed in the following way.

From an ideal triangulation to a protoMom-structure: constructive approach Consider the 3-manifold $M^{\prime}$ obtained by removing from each tetrahedron of $\tau$ a small open ball around the centre of the tetrahedron. The triangulation $\tau$ naturally induces on $M^{\prime}$ a general-based handle decomposition $\Delta^{\prime}$ obtained by taking the collar of $\Sigma$ and thickening each edge of $\tau$ to a 1 -handle and each face to a 2-handle.

We can construct a (nonunique) weak protoMom-structure $(M, \Sigma, \Delta)$ with $M \subset M^{\prime}$ and $\Delta$ induced by $\Delta^{\prime}$ by starting from $\Delta^{\prime}$ and repeatedly deleting 2-handles $\alpha$ satisfying one of the following conditions: 
(a) the ends of the cocore of $\alpha$ lie on two distinct spherical components of the boundary, or

(b) one end of the cocore of $\alpha$ lies on a spherical component and the other one on a toric component, or

(c) both ends of the cocore of $\alpha$ lie on the same spherical component.

We do this until a handle decomposition of a manifold bounded by $\Sigma$ and some tori is reached.

Denote the manifold obtained as a result by $M$ and the subset of the handles of $\Delta^{\prime}$ that are contained in $M$ by $\Delta$. Then it is evident that $(M, \Sigma, \Delta)$ thus obtained is a weak protoMom-structure internal on $N$.

Remark 2.1 Observe that the procedure described above involves the minimal possible number of handle deletions required to obtain an internal protoMom-structure composed of handles belonging to $\Delta^{\prime}$ (this fact will be given a precise formulation in Section 4; see Definition 4.8 and Lemma 4.11). Note however that canceling a 1-handle of valence 1 with the 2 -handle incident to it transforms a weak protoMom-structure into a weak protoMom-structure, so performing such cancelations intermittently with the above operation allows us to obtain a much wider class of protoMom-structures from a given triangulation. An a priori still wider class of protoMom-structures could be obtained by allowing the removal of 1 -handles of valence 0 intermittently with the removal of 2-handles, but we will not consider this latter procedure in the paper.

Remark 2.2 We note in particular that, as will easily follow from Lemma 4.13 below, for any "genuine" (ie, nonweak) full protoMom-structure (hence for any Mom-structure considered in $[6 ; 4 ; 5])$ there exist a triangulation and a choice of 2 -handles to remove via the operation described above, such that the subsequent cancelation of 1-handles of valence 1 until none such is left yields the original protoMom-structure.

We now give a formal definition of a notion already referred to above.

Definition 2.3 Let $N$, with $\partial N=\Sigma$, $\tau$, and $\Delta^{\prime}$ be as above. A weak protoMomstructure $(M, \Sigma, \Delta)$ internal on $N$ is said to be $\tau$-induced if the set of handles of $\Delta$ is a subset of that of $\Delta^{\prime}$.

Note that, while any protoMom-structure obtained as described above is of course $\tau$-induced, the latter notion is a priori more general. Indeed, suppose that we have a $\tau-$ induced protoMom-structure $(M, \Sigma, \Delta)$ containing a subset of 1-and 2-handles such 
that: (1) the subset forms an annular sheet in the sense of [6]; (2) the lateral boundary of this sheet consists of two annuli belonging to two different lateral boundary components of the ambient manifold $M$; (3) each such lateral annulus is nontrivial in the respective lateral torus. Then removing such sheet still yields a $\tau$-induced protoMom-structure, which may or may not be obtainable via a sequence of the operations described above.

Geometric protoMom-structures and canonical decompositions We briefly observe that a certain adjustment of the above constructive procedure allows to obtain a number of weak protoMom-structures internal on nonclosed hyperbolic 3-manifolds without annular cusps and geometric in the sense of [4; 5] (ie, such that the cores of all the 1 - and the 2-handles are respectively geodesic arcs and geodesic hexagons). The adjustment is as follows. We consider, as appropriate, either the Epstein-=Penner [2] or the Kojima decomposition [8; 9] of $N$, and we subdivide each face of each polyhedron into triangles by geodesic arcs. Then we construct $\Delta^{\prime}$ by taking thickenings of the edges of the polyhedra and of the added arcs as 1-handles, and the thickenings of the triangles as 2-handles $\left(M^{\prime}\right.$ is then the ambient space of $\Delta^{\prime}$ ), and we apply precisely the same procedure as above (with or without the collapses) to obtain a geometric weak protoMom-structure from $\Delta^{\prime}$.

\subsection{From an internal protoMom-structure to an ideal triangulation}

In this section we will establish the following result.

Theorem 2.4 Let $(M, \Sigma, \Delta)$ be a full weak protoMom-structure internal on a compact connected orientable 3-manifold $N$ with $\partial N=\Sigma$. Then there exists an ideal triangulation $\tau$ of $N$ such that $(M, \Sigma, \Delta)$ is $\tau$-induced.

To prove this theorem, we will need several auxiliary tools, including one well-known construction described in the next paragraph.

Layered triangulations Given a 3-manifold $M^{\prime}$ with a toric boundary component $T$ triangulated into two triangles, a layering along an edge e (see Jaco, Rubinstein and Tillmann [7] and the references therein) of this triangulation consists in gluing a standard tetrahedron $\Delta_{3}$ to $T$ via a simplicial homeomorphism between $\alpha \cup \beta$, where $\alpha$ and $\beta$ are the two triangular faces in the triangulation of $T$ (and are therefore adjacent to $e$ ), and two faces $\alpha^{\prime}$ and $\beta^{\prime}$ in the boundary of $\Delta_{3}$, such that the common edge $\alpha^{\prime} \cap \beta^{\prime}$ is glued to $e$. Clearly, this operation changes the triangulation of $T \subset \partial M^{\prime}$ but not the manifold $M^{\prime}$ itself; we will need it to describe the so-called layered triangulations of solid tori. 

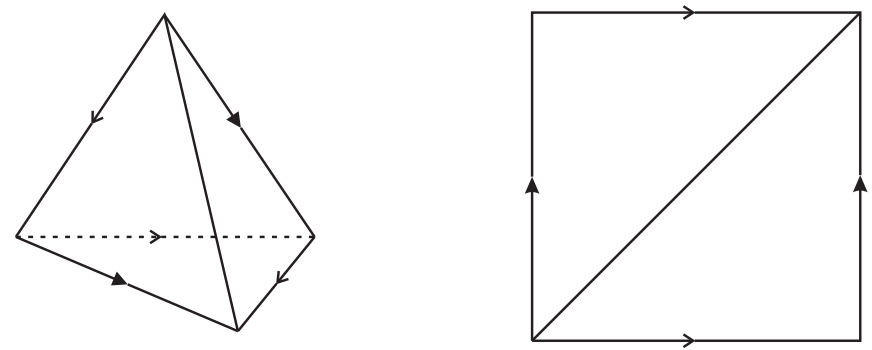

Figure 3. Left: one-tetrahedron triangulation of a solid torus. The two back faces are glued together according to the labels of the edges. Right: the resulting triangulation of the boundary torus.

A layered triangulation of a solid torus $H$ is any triangulation of $H$ obtained by taking the one-tetrahedron triangulation of $H$ shown in Figure 3(left) and then performing several successive operations of layering along edges in $\partial H$. If $\tau$ is a layered triangulation of $H$ then its restriction to the boundary of $H$ is always of the form shown in Figure 3(right); the operation of layering changes the position of this triangulation with respect to the meridional disc of $H$. More precisely, the set of triangulations of $\partial H$ as in Figure 3(right) is in bijective correspondence with the set of isotopy classes of embeddings of the $\theta$-curve in $\partial H$ having a disc as the complement, where the bijection is by taking the dual graph to the 1-skeleton of the triangulation, as in Figure 4.

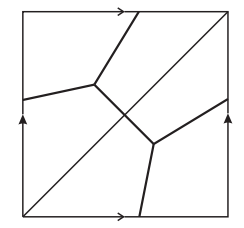

Figure 4. The dual $\theta$-curve

To proceed, we will need the following known result.

Lemma 2.5 [7] For every $\theta$-curve in the boundary of a solid torus $H$ having a disc as the complement there exists a layered triangulation of $H$ such that the 1-skeleton of its restriction to $\partial H$ is dual to the $\theta$-curve.

Induced triangulations of lateral tori Observe that a full weak protoMom-structure cannot contain 1-handles of valence 0 . Hence, each lateral torus $T_{i}$ of the ambient manifold $M$ of such a protoMom-structure admits a natural decomposition into disks of the following 3 types: 
- lakes (contained in $\Sigma \times\{1\}$ );

- strips of the form $\ell \times[0,1]$ with $\ell \subset \partial D^{2}$ for some 1 -handle $D^{2} \times[0,1]$;

- hexagons that are lateral sides of 2-handles.

This decomposition induces a natural triangulation of $T_{i}$ obtained by compressing each lake to a point and each strip $\ell \times[0,1]$ to an $\operatorname{arc}\{*\} \times[0,1]$. We denote this triangulation by $\delta^{\prime}\left(T_{i}\right)$.

Moves on triangulations of surfaces We now introduce several moves on triangulations of surfaces, that will be used to prove Theorem 2.4. Let $T$ be a closed surface endowed with a triangulation $\delta$.

(s1) Let $v$ be a vertex of $\delta$, and let $e^{\prime}, e^{\prime \prime}$ be two distinct edges incident to $v$. Cut $T$ open along $e_{1} \cup v \cup e_{2}$ and fill the resulting square by two triangles sharing an edge in the original position of $e_{1} \cup v \cup e_{2}$. We say that the move (s1) is performed at $v$ along $e^{\prime}, e^{\prime \prime}$.

(s2) Let $e$ be an edge of $\delta$ belonging to two distinct triangles. Remove $e$ and replace it by the other diagonal $e^{\prime}$ of the resulting square ("flipping" $e$ ). We say that the move (s2) is performed at e.

(s3) Let $v$ be a vertex of $\delta$ incident to exactly 3 distinct triangles. Merge the three triangles into a single one, removing $v$ and the edges incident to it.

The moves (s1)-(s3) are shown in Figure 5.
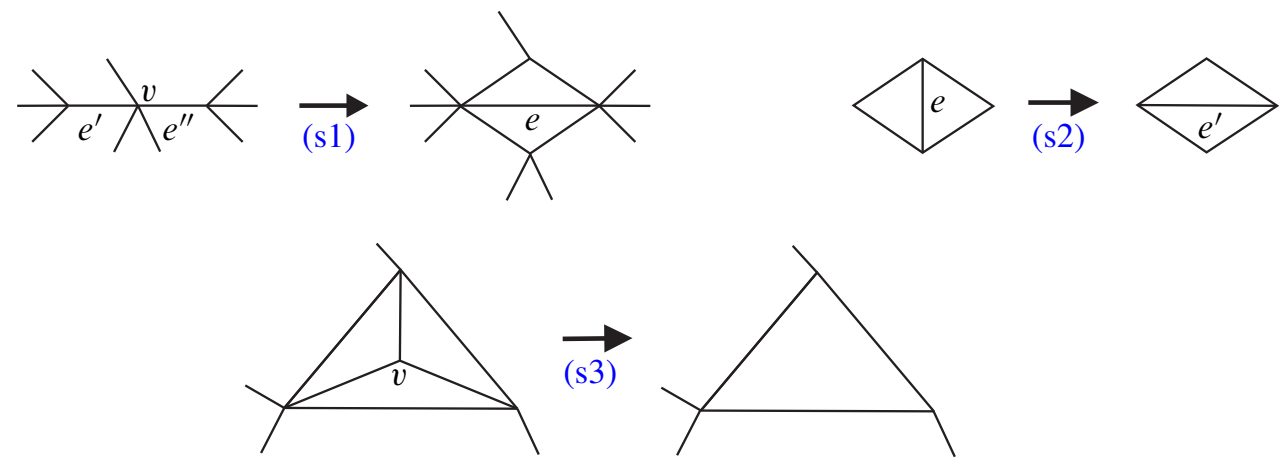

Figure 5. The moves (s1), (s2), (s3)

We will most often apply the move (s1) at a vertex $v^{\prime}$ along two edges $e^{\prime}, e^{\prime \prime}$ such that $e^{\prime}$ is incident to a vertex $v$ of valence 1 ; see Figure 6 . Observe that the move destroys $v$ without creating vertices of valence 1 or 2 . 


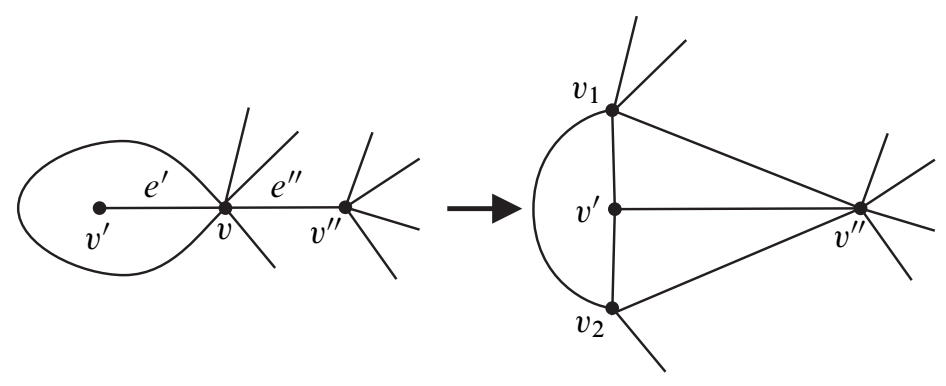

Figure 6. A special case of move (s1)

Below we will often use the following composition of moves.

$\left(\mathrm{s} 1^{\prime}\right)$ Let $v$ be a vertex of $\delta$ of valence 2. Denote the two edges incident to $v$ by $e^{\prime}$ and $e^{\prime \prime}$. The move $\left(\mathrm{s}^{\prime}\right)$ consists in performing the move (s1) at $v$ along $e^{\prime}, e^{\prime \prime}$ and subsequently performing the move (s2) along the newly created edge $e$.

The effect of this move on the triangulation is shown in Figure 7. Observe that the

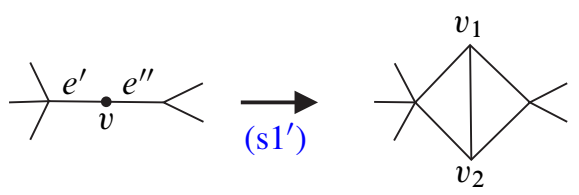

Figure 7. The move (s1')

move decreases the number of vertices of valence 2 by one, and does not create any vertices of valence 1 .

Simplifying a triangulation The key ingredient in the proof of Theorem 2.4 will be the following fact.

Lemma 2.6 Let $\delta$ be a triangulation of a two-dimensional torus. Then there exists a sequence of moves (s1), (s2), (s3) transforming $\delta$ into a triangulation with exactly two triangles.

The desired triangulation is the one shown in Figure 3(right).

Proof The strategy of the proof is to use first the moves $(s \star)$ to get rid of all vertices of valence 1 and 2 ; note that, since we will use the move $\left(\mathrm{s}^{\prime}\right)$, this may increase the total number of vertices. We then show that a triangulation where all vertices have 
valence at least 3, can be transformed into the desired one by moves (s2) and (s3), and the argument is based on the fact that the move (s3) strictly decreases the number of vertices.

Case 1 Assume first that $\delta$ contains a vertex $v$ of valence 1 . Then it is easy to see that we have a situation as in Figure 8(left), possibly with $v_{1}=v_{2}$. We then perform

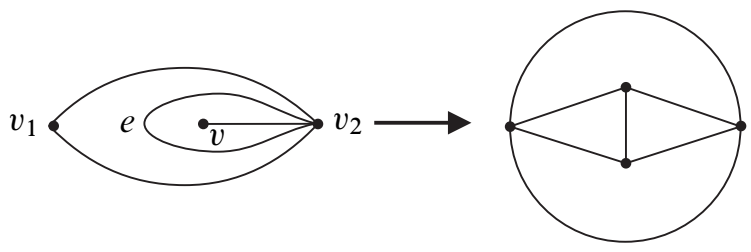

Figure 8 . Destroying a vertex of valence 1 ; note that $v_{1}$ and $v_{2}$ may coincide.

the move (s2) at the edge $e$, followed by the move $\left(\mathrm{s} 1^{\prime}\right)$ at the vertex $v$, as shown in Figure 8. This destroys the vertex $v$ of valence 1 without creating new vertices of valence 1 or 2 , so we can repeat the procedure until no vertices of valence 1 are left.

Case 2 Assume now that $\delta$ has a vertex $v$ of valence 2 but not of valence 1 . We then perform $\left(\mathrm{s} 1^{\prime}\right)$ at $v$ and proceed until no vertex of valence 1 or 2 remains.

Case 3 Assume now that all vertices of $\delta$ have valence at least 3 . The proof is by induction on the number $k$ of vertices of $\delta$. The base of induction is $k=1$ and it follows from the Euler characteristic argument that in this case we indeed have the triangulation with two triangles. Assume that $k>1$.

Case 3.1 Suppose that $\delta$ contains a vertex $v$ of valence precisely 3 . It is easy to see that in this case the closure of any edge incident to $v$ is a segment. There are now the following two possibilities:

Case 3.1.a There exists a vertex $v$ of valence 3 such that the three vertices $v_{1}, v_{2}, v_{3}$ joined to $v$ by an edge are all of valence at least 4 . Observe first that if two of these vertices, say $v_{2}$ and $v_{3}$, coincide then the valence of $w=v_{2}=v_{3}$ is at least 5; furthermore, if all three vertices coincide then the valence of $w=v_{1}=v_{2}=v_{3}$ is at least 7 . We now perform the move (s3) at $v$, the effect of doing which is that $v$ disappears and the valences of $v_{1}, v_{2}, v_{3}$ are decreased by 1 , if they are all distinct; if they are not, the valence of $w$ is decreased by 2 or by 3 , depending on how many of the vertices $v_{i}$ are different. In either case, by the assumption these new valences are still at least 3 . In addition, observe that the valences of all the other vertices are not affected, and that the total number of vertices in $\delta$ is decreased by 1 . 
Case 3.1.b The second possibility is that for any vertex $v$ of valence 3 at least one of the vertices $v_{1}, v_{2}, v_{3}$ joined to $v$ by an edge has valence 3 , with $v_{i}$ 's not necessarily all distinct. Choose any such $v$ and observe that we must have the situation as shown in Figure 9, with the valences of $v_{2}, v_{3}$ being at least 5 and where we may or may not

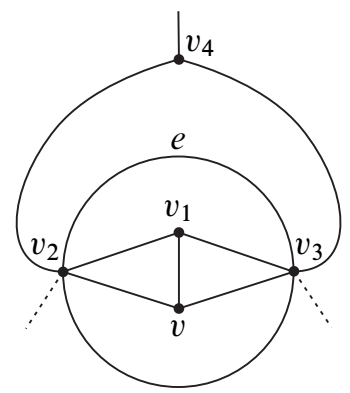

Figure 9. Two vertices of valence 3 joined by an edge

have $v_{2}=v_{3}$. Denote by $e$ one of the edges joining $v_{2}$ to $v_{3}$; then $e$ belongs to two triangles, one with vertices $v_{1}, v_{2}, v_{3}$ and the other with vertices $v_{2}, v_{3}, v_{4}$; we also have that $v_{4} \neq v_{1}$. Thus, performing the move (s2) at $e$ has the following effect on the set of valences:

- the valence of $v_{1}$ becomes 4 ;

- the valence of $v_{4}$ is increased by 1 ;

- if $v_{2} \neq v_{3}$ then the valence of each of them decreases by 1 , and if they coincide then the total valence is decreased by 2 , however, in this case it was at least 6 to begin with.

Notice that no other vertices are affected by the move and that after the move the valences of $v_{2}$ and $v_{3}$ (or that of $v_{2}=v_{3}$ ) are still at least 4 . Therefore we can proceed as in Case 3.1.a.

Case 3.2 Suppose that all vertices of $\delta$ have valence at least 4. Again, there are two possibilities:

Case 3.2.a There is a vertex such that the closure of any edge incident to it is a segment. Let $v$ be a vertex of minimal valence among all such vertices. Denote the edges incident to $v$ by $e_{1}, \ldots, e_{k}$ in the clockwise order around $v$ and let $v_{i}$ be the other endpoint of $e_{i}$ for $i=1, \ldots, k$ (we note that some of the $v_{i}$ 's may coincide). Perform now the move (s2) consecutively along the edges $e_{2}, e_{3}, \ldots, e_{k-2}$, followed by the move (s3) at the vertex $v$; see Figure 10. Observe that 

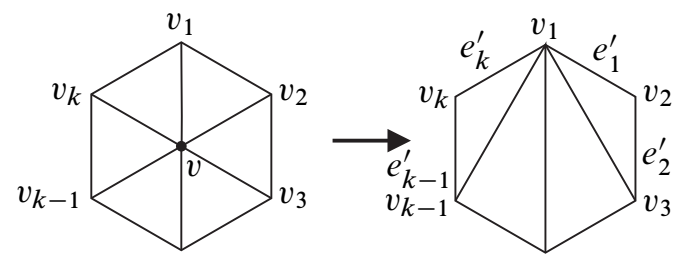

Figure 10. Destroying vertex $v$

- the valence of any vertex (among $v_{i}$ 's) distinct from $v_{2}$ and $v_{k}$ does not decrease;

- if $v_{2} \neq v_{k}$ then the valence of each of these vertices is decreased at most by 1 , hence it is still at least 3 .

Suppose now that $v_{2}=v_{k}=w$, and that the valence of $w$ is at most 2; this implies in particular that $w \neq v_{1}$ and that the valence of $w$ before the move was at most 4 . We claim that in this case $w$ cannot be incident to a loop; indeed, if it were, the loop edge would be distinct from the edge $e_{1}^{\prime}$, hence after the moves the valence of $w$ would be at least 3 . Now, since $v$ was a vertex of minimal valence among those edges not incident to any loop, this implies that $k=4$ and that among the edges $e_{1}^{\prime}, e_{2}^{\prime}, e_{3}^{\prime}, e_{4}^{\prime}$ there are precisely two distinct ones. However, this would imply that we already have a triangulation of the torus into two triangles.

Since the total number of vertices diminishes, we can repeat the entire procedure, starting from Case 3, until we either reach a triangulation with exactly two triangles or encounter the situation as in Case 3.2.b below.

Case 3.2.b Suppose that all vertices are incident to at least one edge whose closure is a loop. In this case all such loops are nontrivial in $T$ because if one of them were the boundary of a disc, the assumption just made implies that an innermost loop in this disc would contradict the fact that we are dealing with a triangulation. Then one can see directly that, up to applying the move (s2), we have a situation as shown in Figure 11 (more precisely, the triangulation either will be exactly as in the figure or
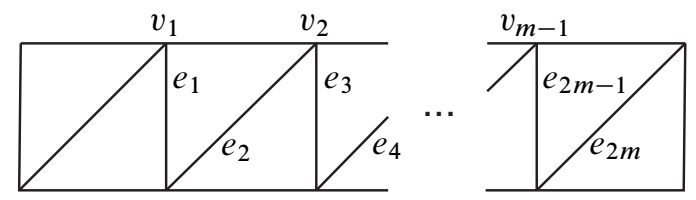

Figure 11. A series of particular triangulations of a torus; the opposite sides of the rectangle are identified forming the torus.

can be obtained from it by performing (s2) at the diagonals of some of the squares). In this case the proof is by induction on the number $m$ of parallel loops. 
If $m=1$, we already have the desired triangulation. If $m>1$ then we perform the move (s2) at $e_{1}$, then (s2) at $e_{2}$, and finally (s3) at $v_{1}$. This again yields a triangulation as in Case 3.2.b but the number $m$ decreases by 1, whence the conclusion.

Constructing triangulations of solid tori Let now $H$ be a solid torus, and let $\delta$ be a triangulation of $T=\partial H$. We define the following moves consisting in inserting inside $H$ some 2 - or 3-dimensional simplices.

( $\hat{\mathrm{s}} 1)$ Let $v$ be a vertex of $\delta$, and let $e^{\prime}, e^{\prime \prime}$ be two distinct edges adjacent to $v$. The move ( $\hat{\mathrm{s}} 1)$ consists in gluing to $T$, inside $H$, a triangle via a piecewise-linear identification of two of its edges with $e^{\prime} \cup v \cup e^{\prime \prime}$. We say that the move ( $\left.\hat{\mathrm{s}} 1\right)$ is performed at $v$ along $e^{\prime}, e^{\prime \prime}$.

( $\hat{\mathrm{s}} 2)$ Let $e$ be an edge of $\delta$ adjacent to two distinct faces $\alpha$ and $\beta$. The move ( $\hat{\mathrm{s}} 2)$ consists in gluing to $T$, inside $H$, a tetrahedron via a piecewise-linear identification of two of its faces with $\alpha \cup \beta$. We say that the move ( $\hat{\mathrm{s}} 2)$ is performed at $e$; note that this is the same operation as the one performed in the construction of any layered triangulation.

( $\hat{\mathrm{s}} 3$ ) Let $v$ be a vertex of $\delta$ incident to exactly 3 distinct edges. Denote the three triangles incident to $v$ by $\alpha_{1}, \alpha_{2}, \alpha_{3}$. The move ( $\hat{\mathrm{s}} 3$ ) consists in gluing to $T$, inside $H$, a tetrahedron via a piecewise-linear identification of three of its faces with $\alpha_{1} \cup \alpha_{2} \cup \alpha_{3}$.

We also define the following composite move.

$\left(\hat{\mathrm{s}} 1^{\prime}\right)$ Let $v$ be a vertex of $\delta$ of valence 2 . Denote the two edges adjacent to $v$ by $e^{\prime}$ and $e^{\prime \prime}$. The move $\left(\hat{\mathrm{s}} 1^{\prime}\right)$ consists in performing move $(\hat{\mathrm{s}} 1)$ at $v$ along $e^{\prime}, e^{\prime \prime}$ and subsequently performing move ( $\hat{\mathrm{s}} 2$ ) along the newly created edge.

There is a natural correspondence between the moves $(\mathrm{s} \star)$ and $(\hat{\mathrm{s}} \star)$ described in the following:

Observation 2.7 Let $H$ be a solid torus, and let $\delta$ be a triangulation of $T=\partial H$. Let $\gamma$ be the simplex added by a move ( $\hat{\mathrm{S}} \star$ ) applied to $H$, and let $\delta^{\prime}$ be the induced triangulation of the surface $T^{\prime}$, where $T^{\prime}$ is the connected component of $\partial U(T \cup \gamma)$ that lies inside $H$, with $U(X)$ denoting a regular neighbourhood, in $H$, of any subcomplex $X$ of $H$. Then $\delta^{\prime}$ is obtained from $\delta$ by a move $(\mathrm{s} \star)$.

We can now establish the following:

Lemma 2.8 Let $\delta$ be a triangulation of a two-dimensional torus $T=\partial H$, where $H$ is a solid torus. Then there exists a triangulation $\tau$ of $H$ such that $\left.\tau\right|_{\partial H}=\delta$, and $\tau^{(0)}=\delta^{(0)}$. 
Proof We construct the desired triangulation by following the proof of Lemma 2.6 and performing for each move $(s \star)$ the corresponding move $(\hat{\mathrm{s}} \star)$. Here the surface that tells us which move to apply, is, at each step, the connected component of the boundary of $U\left(T \cup\left\{\gamma_{i}\right\}\right)$ (where $\gamma_{i}$ are all the simplices added by the moves ( $\left.\hat{s} \star\right)$ already performed) that lies in the interior of $H$ and is endowed with the obvious triangulation, whereas the simplices themselves are glued, inside $H$, to the simplicial complex $T \cup\left\{\gamma_{i}\right\}$. By Lemma 2.6 we will end up with a solid torus $H^{\prime} \subset H$ (possibly with immersed boundary) such that $\partial H^{\prime}$ is triangulated into two triangles (while $H \backslash$ Int $H^{\prime}$ is already triangulated in a desired fashion, ie such that all the vertices are in $\partial H$ ), and we conclude the proof by adding a suitable layered triangulation.

Proof of Theorem 2.4 Denote the lateral tori of $(M, \Sigma, \Delta)$ by $T_{1}, \ldots, T_{k}$ and consider the triangulations $\delta^{\prime}\left(T_{i}\right)$ induced by $\Delta$, as described at the beginning of this section. Let $H_{i}$ be the solid torus bounded by $T_{i}$ in $N$. For each $i=1, \ldots, k$ denote by $\tau_{i}$ the triangulation of $H_{i}$ provided by Lemma 2.8 applied to $T_{i}=\partial H_{i}$ and $\delta^{\prime}\left(T_{i}\right)$. It now follows directly from the construction that the triangulations $\tau_{i}, i=1, \ldots, k$, yield an ideal triangulation $\tau$ of $N$ (we emphasize that the triangulation is an ideal one because its vertices, obtained by contracting the lakes of the $T_{i}$ 's, all lie on $\Sigma$ ) and that the protoMom-structure $(M, \Sigma, \Delta)$ is $\tau$-induced, whence the conclusion.

\section{Mom-subgraphs in 4-valent graphs}

We will now describe a combinatorial tool that we will use to show that any two internal protoMom-structures on a given $N$ are related by the combinatorial moves that will be described in Section 4.1. This tool is that of a Mom-subgraph; as will be explained in Section 4 (see Lemma 4.11) these objects are dual, in a certain natural sense, to a particular type of triangulation-induced protoMom-structures, the type that will play an especially important role in establishing the rest of our results.

\subsection{Minimal Mom-subgraphs}

The above-mentioned notion of a Mom-subgraph comes in two types, and in this subsection we study the first of them, called a minimal Mom-subgraph.

Definition 3.1 Let $G$ be a connected 4-valent graph. A minimal Mom-subgraph $\Gamma$ of $G$ is a complete coloring of the edges of $G$ by colors $\{t, c, f\}$ such that

- the union $T(\Gamma)$ of the edges with color $t$ gives a maximal tree in $G$;

- precisely one edge, denoted $c(\Gamma)$, has color $c$.

We also define $\hat{T}(\Gamma):=T(\Gamma) \cup c(\Gamma)$, and $C(\Gamma)$ as the only simple closed curve contained in $\widehat{T}(\Gamma)$. Note that $c(\Gamma) \subset C(\Gamma)$. 
Moves on minimal Mom-subgraphs We first describe two admissible moves that can be applied to a minimal Mom-subgraph $\Gamma$ of some $G$.

(m1) if $e=c(\Gamma)$ and some edge $e^{\prime}$ of color $f$ share a vertex $v$, switch the colors of $e$ and $e^{\prime}$;

(m2) if $e \subset T(\Gamma)$ and $e^{\prime} \subset(G \backslash T(\Gamma))$ share a vertex $v$, and $(T(\Gamma) \backslash e) \cup e^{\prime}$ is connected (equivalently, $e$ lies along the path in $T(\Gamma)$ which joins the endpoints of $\left.e^{\prime}\right)$, switch the colors of $e$ and $e^{\prime}$.
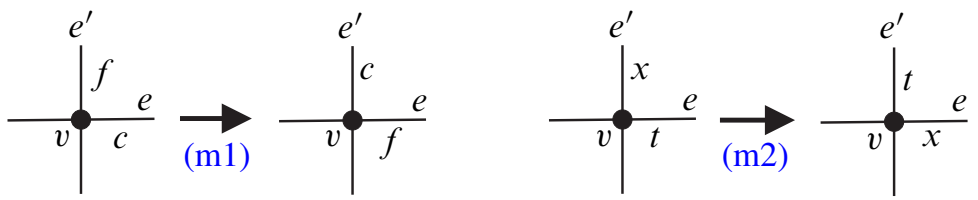

Figure 12. Moves on Mom-subgraphs; on the right, we have $x \in\{c, f\}$.

In both cases we will say that the move is applied at $\left(v, e, e^{\prime}\right)$; see Figure 12. The following statement is obvious.

Lemma 3.2 The moves $(\mathrm{m} 1)$ and $(\mathrm{m} 2)$ transform a minimal Mom-subgraph into another one.

We will mostly use not a single move $(\mathrm{m} 2)$ but a particular composition of several such moves, namely,

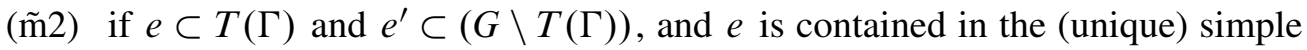
path $\ell$ in $T(\Gamma)$ that joins the endpoints of $e^{\prime}$, switch the colors of $e$ and $e^{\prime}$.

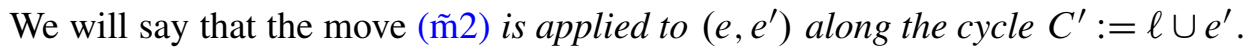

Lemma 3.3 The move ( $\tilde{\mathrm{m}} 2)$ is a composition of moves $(\mathrm{m} 2)$.

Proof Let $\ell^{\prime}$ be one of the connected components of $\ell \backslash \operatorname{Int}(e)$, and let $e_{1} \subset \ell^{\prime}$ be the edge incident to $e^{\prime}$. Denote the endpoints of $e^{\prime}$ by $v^{\prime}=e^{\prime} \cap e_{1}$ and $v^{\prime \prime}$, and denote the other endpoint of $e_{1}$ by $v_{1}$. Observe now that, since $e_{1}$ is separating for $T(\Gamma)$, the vertices $v_{1}$ and $v^{\prime}$ are contained in different connected components of $T(\Gamma) \backslash \operatorname{Int}\left(e_{1}\right)$. Furthermore, the existence of the path $\ell$ implies that $v^{\prime \prime}$ belongs to the same connected component as $v_{1}$, hence we can apply the move $(\mathrm{m} 2)$ at $\left(v^{\prime}, e_{1}, e^{\prime}\right)$. The conclusion then follows by the induction on the number of edges in the fixed $\ell^{\prime}$.

We will refer to the moves ( $\mathrm{m} 1)$ and $(\mathrm{m} 2)$ as to admissible moves; by the above lemma, $(\tilde{\mathrm{m}} 2)$ is a composition of such. 
Relating minimal Mom-subgraphs by the moves We now establish the following result.

Proposition 3.4 Let $G$ be a connected 4-valent graph. Then any two minimal Momsubgraphs $\Gamma_{1}$ and $\Gamma_{2}$ of $G$ are connected by a sequence of admissible moves.

Proof The proof is by induction on the number $n\left(\Gamma_{1}, \Gamma_{2}\right)$ of edges $e$ of $G$ having different colors in $\Gamma_{1}$ and $\Gamma_{2}$ for $e$ belonging to exactly one of $\hat{T}\left(\Gamma_{1}\right)$ or $\hat{T}\left(\Gamma_{2}\right)$. Note that, since $\hat{T}\left(\Gamma_{1}\right)$ and $\hat{T}\left(\Gamma_{2}\right)$ contain the same number of edges, $n\left(\Gamma_{1}, \Gamma_{2}\right)$ cannot be equal to 1 . Furthermore, if $n\left(\Gamma_{1}, \Gamma_{2}\right)=0$ then $\hat{T}\left(\Gamma_{1}\right)=\hat{T}\left(\Gamma_{2}\right)$, therefore $C\left(\Gamma_{1}\right)=C\left(\Gamma_{2}\right)$, and the conclusion is obtained by applying the move ( $\left.\tilde{\mathrm{m}} 2\right)$ at the two $c$-edges along $C\left(\Gamma_{1}\right)$.

Suppose now that $n\left(\Gamma_{1}, \Gamma_{2}\right)>0$. We distinguish two cases.

(1) Assume that $T\left(\Gamma_{1}\right) \neq T\left(\Gamma_{2}\right)$. Since also $\widehat{T}\left(\Gamma_{1}\right) \neq \hat{T}\left(\Gamma_{2}\right)$, we can find an edge $e_{2} \subset\left(T\left(\Gamma_{2}\right) \backslash \hat{T}\left(\Gamma_{1}\right)\right)$, possibly up to change of notation. We now take the only simple closed curve $\ell$ contained in $T\left(\Gamma_{1}\right) \cup e_{2}$; since $T\left(\Gamma_{2}\right)$ is a tree, we can find $e_{1} \subset \ell$

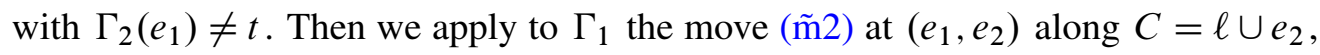
reducing $n\left(\Gamma_{1}, \Gamma_{2}\right)$.

(2) Assume now that $T\left(\Gamma_{1}\right)=T\left(\Gamma_{2}\right)$. This immediately implies that $n\left(\Gamma_{1}, \Gamma_{2}\right)$ is 2 . More precisely, for $e_{i}=c\left(\Gamma_{i}\right)$ with $i=1,2$ we have $\Gamma_{1}\left(e_{2}\right)=f=\Gamma_{2}\left(e_{1}\right)$, while any other edge is assigned the same color by both $\Gamma_{1}$ and $\Gamma_{2}$.

The proof is by induction on the distance $k$ between $C\left(\Gamma_{1}\right)$ and $C\left(\Gamma_{2}\right)$. Namely, let $\ell$ be the shortest path in $T\left(\Gamma_{1}\right)$ joining $C\left(\Gamma_{1}\right)$ to $C\left(\Gamma_{2}\right)$; we define $\ell$ to be empty if $C\left(\Gamma_{1}\right) \cap C\left(\Gamma_{2}\right)$ contains at least one edge. Note that if $C\left(\Gamma_{1}\right) \cap C\left(\Gamma_{2}\right)$ consists of a single vertex $v$ then $\ell$ coincides with $v$. The distance $k$ is defined to be the simplicial length of $\ell$, ie the number of edges in $\ell$; by definition, the length of the empty path is $-\infty$.

Base of induction: $k \leqslant 0$. Then $C\left(\Gamma_{1}\right) \cap C\left(\Gamma_{2}\right)$ is a nonempty path in $T\left(\Gamma_{1}\right)=T\left(\Gamma_{2}\right)$; let $v$ be an end vertex of this path (note that we might have $C\left(\Gamma_{1}\right) \cap C\left(\Gamma_{2}\right)=v$ ). Denote by $e^{(1)}$ (one of) the extremal edge(s) of $C\left(\Gamma_{1}\right) \backslash C\left(\Gamma_{2}\right)$ incident to $v$, and by $e^{(2)}$ (one of) the extremal edge(s) of $C\left(\Gamma_{2}\right) \backslash C\left(\Gamma_{1}\right)$ incident to $v$. Apply now to $\Gamma_{1}$ the following sequence of moves:

- $(\tilde{\mathrm{m}} 2)$ at $\left(e_{1}, e^{(1)}\right)$ along $C\left(\Gamma_{1}\right)$;

- $(\tilde{\mathrm{m}} 2)$ at $\left(e_{2}, e^{(2)}\right)$ along $C\left(\Gamma_{2}\right)$;

- (m1) at $\left(v, e^{(1)}, e^{(2)}\right)$;

- $(\tilde{\mathrm{m}} 2)$ at $\left(e_{2}, e^{(2)}\right)$ along $C\left(\Gamma_{2}\right)$;

- $(\tilde{\mathrm{m}} 2)$ at $\left(e_{1}, e^{(1)}\right)$ along $C\left(\Gamma_{1}\right)$. 
It is then easy to see that the resulting coloring is in fact $\Gamma_{2}$. It follows from Lemma 3.3 that all of the above operations are compositions of admissible moves, whence the conclusion in this case.

Inductive step Suppose that $k>0$; then $\ell$ contains at least one edge. Let $w$ be the vertex at which $\ell$ meets $C\left(\Gamma_{1}\right)$, and let $T^{\prime}$ be the connected component of $T\left(\Gamma_{1}\right) \backslash w$ containing $\ell$; notice that $T^{\prime}$ is not compact. We claim that there exists an $f$-edge $e$ with one endpoint in $T^{\prime}$ and the other not belonging to $T^{\prime}$ (but possibly coinciding with $w$ ). Indeed, assume that such an edge does not exist. Then all $f$-edges with one endpoint on $T^{\prime}$ would have the other endpoint on $T^{\prime}$ as well, so the obvious compactification of $\left(T^{\prime} \cup\left\{f\right.\right.$ - edges with an endpoint on $\left.\left.T^{\prime}\right\} \cup e_{2}\right)$ would be a graph with one vertex of valence 1 and all the other vertices of valence 4 , which is impossible.

Consider now an edge $e$ with the above property, and let $\ell^{\prime}$ be the simple path in $T\left(\Gamma_{1}\right)$ joining its endpoints. Observe that $\ell^{\prime}$ contains $w$ and has at least one edge in common with $\ell$. Denote by $e^{(1)}$ an edge of $C\left(\Gamma_{1}\right)$ incident to $w$ and not contained in $\ell^{\prime}$, and by $e^{(2)}$ the edge of $\ell$ incident to $w$; notice that $e^{(2)}$ is contained in $\ell^{\prime}$. Apply now to $\Gamma_{1}$ the following moves:

- $(\tilde{\mathrm{m}} 2)$ at $\left(e_{1}, e^{(1)}\right)$ along $C\left(\Gamma_{1}\right)$;

- $(\tilde{\mathrm{m}} 2)$ at $\left(e, e^{(2)}\right)$ along $\ell^{\prime} \cup e$;

- $(\mathrm{m} 1)$ at $\left(w, e^{(1)}, e^{(2)}\right)$;

- $(\tilde{\mathrm{m}} 2)$ at $\left(e, e^{(2)}\right)$ along $\ell^{\prime} \cup e$;

- $(\tilde{\mathrm{m}} 2)$ at $\left(e_{1}, e^{(1)}\right)$ along $C\left(\Gamma_{1}\right)$.

Denote the resulting coloring by $\Gamma_{1}^{\prime}$. Observe that $\Gamma_{1}^{\prime}$ and $\Gamma_{2}$ are still related as in case (2), and that the distance between $C\left(\Gamma_{1}^{\prime}\right)$ and $C\left(\Gamma_{2}\right)$ is strictly less than that between $C\left(\Gamma_{1}\right)$ and $C\left(\Gamma_{2}\right)$, and this concludes the inductive step.

Remark 3.5 In what follows (Proposition 4.12) we will only use a weaker form of Proposition 3.4, namely the fact that given $\Gamma_{1}, \Gamma_{2}$ one can use (m1) and (m2) to transform $\Gamma_{1}$ into some $\Gamma_{1}^{\prime}$ such that $\widehat{T}\left(\Gamma_{1}^{\prime}\right)=\widehat{T}\left(\Gamma_{2}\right)$, but for the sake of completeness we present the above stronger version.

\subsection{General Mom-subgraphs}

In this subsection we consider a more general instance of Mom-subgraphs than minimal ones:

Definition 3.6 Let $G$ be a connected 4-valent graph. A general Mom-subgraph $\Gamma$ in $G$ is a complete coloring of the edges of $G$ by colors $\{t, c, f\}$ such that 
- the set $T(\Gamma)$ of the edges of $G$ that have color $t$ spans $G$;

- each connected component of $T(\Gamma)$ is a tree;

- each edge colored by $c$ has both endpoints on the same connected component of $T(\Gamma)$, and for each connected component of $T(\Gamma)$ there is precisely one $c$-colored edge with vertices on it.

Note that the set of $c$-edges is in bijective correspondence with the set of connected components of $T(\Gamma)$.

For the rest of this subsection we fix the following notation. If $\Gamma$ is a general Momsubgraph of some $G$ then

- $k_{\Gamma}$ is the number of connected components of $T(\Gamma)$;

- $T_{i}(\Gamma)$ with $i=1, \ldots, k_{\Gamma}$ are the connected components of $T(\Gamma)$;

- $e_{i}$ is the $c$-edge with the endpoints on $T_{i}(\Gamma)$;

- $C_{i}(\Gamma)$ is the unique simple cycle in $T_{i}(\Gamma) \cup e_{i}$.

Moves on general Mom-subgraphs The definition of the moves (m1), (m2) and of the composite move ( $\tilde{\mathrm{m}} 2)$ naturally extends to the context of general Mom-subgraphs. The extension is verbatim for $(\mathrm{m} 1)$, whereas for $(\mathrm{m} 2)$ and $(\tilde{\mathrm{m}} 2)$ we add the requirement that both endpoints of the edge $e^{\prime}$ participating in the move belong to the same component $T_{i}(\Gamma)$. In addition to these, we will also consider three new moves:

$\left(\mathrm{m} 2^{\prime}\right)$ let $e$ be an $f$-edge with one endpoint on $T_{i}(\Gamma)$ and the other on $T_{j}(\Gamma) \backslash C_{j}(\Gamma)$ with $i \neq j$, and let $e^{\prime} \subset T_{j}(\Gamma) \backslash C_{j}(\Gamma)$ be an edge incident to $e$ at a vertex $v$. Assume furthermore that $v$ and $C_{j}(\Gamma)$ belong to different connected components of $\left(T_{j}(\Gamma) \cup C_{j}(\Gamma)\right) \backslash \operatorname{Int}\left(e^{\prime}\right)$. Then switch the colors of $e$ and $e^{\prime}$;

(m3) let $e$ be an $f$-edge with one endpoint on $T_{i}(\Gamma)$ incident to some $e_{j}$ with $j \neq i$ (recall that $e_{j}$ is a $c$-edge). Then assign color $t$ to $e$ and color $f$ to $e_{j}$;

( $\overline{\mathrm{m}} 3)$ let $e$ be an edge in $T_{i}(\Gamma)$, and let $e^{\prime}$ be an $f$-edge incident to $e$ and such that both endpoints of $e^{\prime}$ belong to the same connected component of $T_{i}(\Gamma) \backslash \operatorname{Int}(e)$ and this component is not incident to the $c$-edge $e_{i}$. Then assign color $f$ to $e$ and color $c$ to $e^{\prime}$.

The moves are shown in Figure 13. We will say that $\left(\mathrm{m} 2^{\prime}\right)$ and $(\overline{\mathrm{m}} 3)$ are applied at $\left(e, e^{\prime}\right)$ and that $(\mathrm{m} 3)$ is applied at $\left(e, e_{j}\right)$. The next result is now readily established.

Lemma 3.7 We have the following:

(1) The moves $\left(\mathrm{m} 2^{\prime}\right),(\mathrm{m} 3)$, and ( $\left.\overline{\mathrm{m}} 3\right)$ transform any general Mom-subgraph into a general Mom-subgraph.

(2) The inverse of a move $\left(\mathrm{m} 2^{\prime}\right)$ is a move $\left(\mathrm{m} 2^{\prime}\right)$.

(3) The inverse of a move (m3) is a move ( $(\overline{\mathrm{m}} 3)$ and vice versa. 


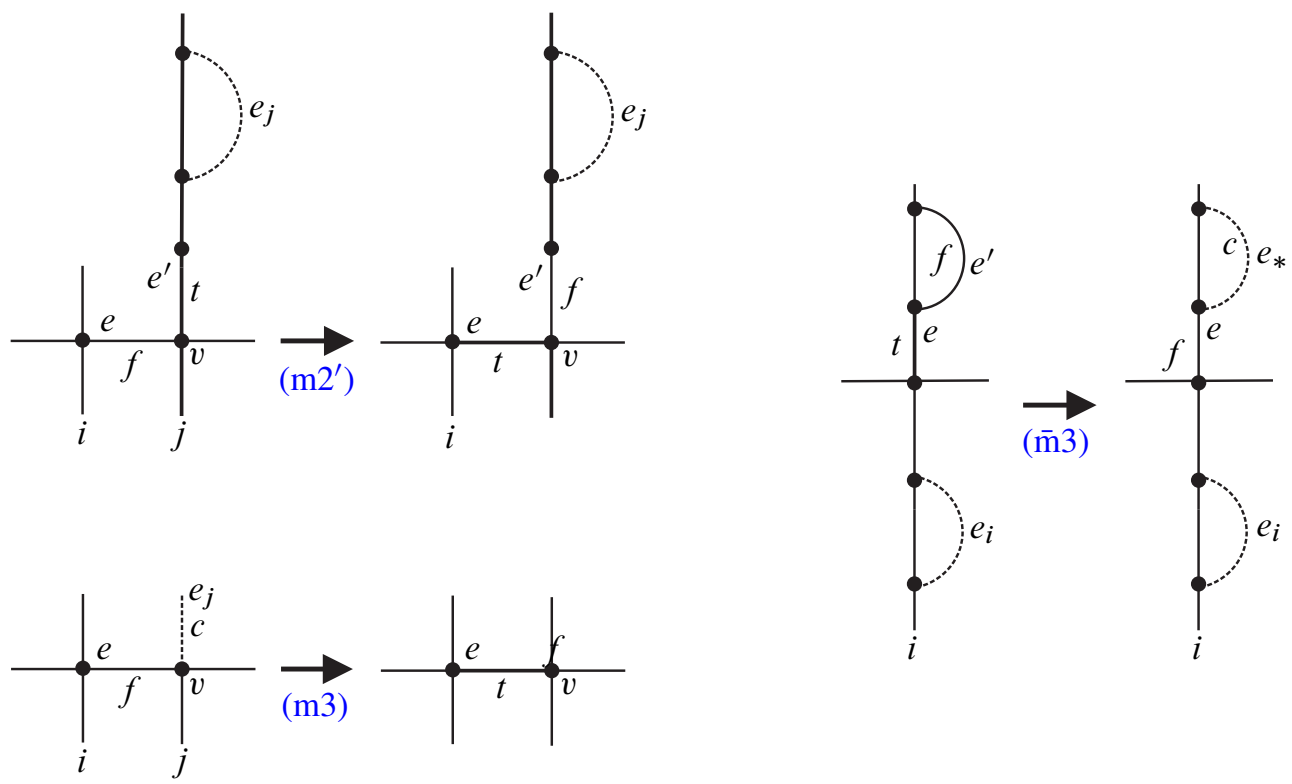

Figure 13. The moves $\left(\mathrm{m} 2^{\prime}\right),(\mathrm{m} 3)$, and $(\overline{\mathrm{m}} 3)$ on general Mom-subgraphs

For brevity, we will refer to the moves $(\mathrm{m} 1),(\mathrm{m} 2),\left(\mathrm{m} 2^{\prime}\right),(\mathrm{m} 3),(\overline{\mathrm{m}} 3)$ as to admissible moves.

Relating general Mom-subgraphs by the moves We now prove the following technical result.

Proposition 3.8 Let $G$ be a connected 4-valent graph. Then every general Momsubgraph $\Gamma$ of $G$ can be transformed to a minimal Mom-subgraph of $G$ via a sequence of admissible moves.

Proof We proceed by induction on $k_{\Gamma}$. The base of the induction is $k_{\Gamma}=1$, in which case $\Gamma$ is already a minimal Mom-subgraph, so there is nothing to prove.

Suppose that $k_{\Gamma}>1$. Since $G$ is connected, there exists an edge of $G$ joining two distinct connected components of $T(\Gamma)$, say $T_{1}(\Gamma)$ and $T_{2}(\Gamma)$. For each such edge $e$ we can define $m_{i}(e)$ as the minimal edge-length of a path in $T_{i}(\Gamma)$ connecting the vertex $e \cap T_{i}(\Gamma)$ to $C_{i}(\Gamma)$; if $e$ is incident to $C_{i}(\Gamma)$ then the path consists of one vertex only and its length is 0 . Let $m(e)=\min \left\{m_{1}(e), m_{2}(e)\right\}$, and let $m_{\Gamma}=\min \{m(e)\}$, where the minimum is taken over all edges connecting $T_{1}(\Gamma)$ to $T_{2}(\Gamma)$. We will show by induction on $m_{\Gamma}$ that there is a sequence of admissible moves transforming $\Gamma$ to a general Mom-subgraph $\Gamma^{\prime}$ such that $k_{\Gamma^{\prime}}=k_{\Gamma}-1$. 
Base of induction: $m_{\Gamma}=0$. This implies that there exists an edge $e$ that has an endpoint on at least one of $C_{1}(\Gamma), C_{2}(\Gamma)$. Up to change of notation, we can assume that $e$ has an endpoint on $C_{2}(\Gamma)$.

If $e$ is incident to $e_{2}$ then the desired $\Gamma^{\prime}$ is obtained by applying (m3) at $\left(e, e_{2}\right)$. Otherwise, denote by $e^{\prime}$ an edge of $C_{2}(\Gamma)$ incident to $e$. Then $\Gamma^{\prime}$ is obtained by applying first the move $(\tilde{\mathrm{m}} 2)$ at $\left(e^{\prime}, e_{2}\right)$ along $C_{2}(\Gamma)$ and then applying $(\mathrm{m} 3)$ at $\left(e, e^{\prime}\right)$. Inductive step. Suppose that $m_{\Gamma}>0$, and let $e$ be an $f$-edge realizing $m_{\Gamma}$. Up to change of notation we can assume that $m_{\Gamma}=m_{2}(e)$. Set $v_{i}=e \cap T_{i}(\Gamma)$.

Let $\ell$ be the shortest path in $T_{2}(\Gamma)$ joining $v_{2}$ to $C_{2}(\Gamma)$, and let $e^{\prime}$ be the edge of $\ell$ incident to $v_{2}$. Apply the move $\left(\mathrm{m} 2^{\prime}\right)$ at $\left(e, e^{\prime}\right)$ and denote the resulting general Mom-subgraph by $\Gamma_{1}$. Notice that $k_{\Gamma_{1}}=k_{\Gamma}$; let $T_{1}\left(\Gamma_{1}\right)$ be the connected component of $T\left(\Gamma_{1}\right)$ containing $T_{1}(\Gamma)$, and let $T_{2}\left(\Gamma_{1}\right)$ be the component containing $C_{2}(\Gamma)$.

Observe now that $T_{1}\left(\Gamma_{1}\right)$ and $T_{2}\left(\Gamma_{1}\right)$ are still joined by an $f$-edge (for example, $e^{\prime}$ ). Moreover, $\ell \backslash e^{\prime}$ joins $e^{\prime} \cap T_{2}\left(\Gamma_{1}\right)$ to $C_{2}(\Gamma)=C_{2}\left(\Gamma_{1}\right)$. Hence $m_{\Gamma_{1}}<m_{\Gamma}$, which concludes both inductions.

We can now easily establish the main result of this section.

Corollary 3.9 Let $G$ be a connected 4-valent graph. Then any two general Momsubgraphs in $G$ are related by a sequence of moves $(\mathrm{m} 1),(\mathrm{m} 2),\left(\mathrm{m} 2^{\prime}\right),(\mathrm{m} 3),(\overline{\mathrm{m}} 3)$.

\section{Moves on protoMom-structures}

In this section we will state and prove the main result of the paper. In the first subsection we introduce two types of combinatorial moves on weak protoMom-structures, and in the second subsection we show that these moves are sufficient to relate to each other any two full weak protoMom-structures internal on the same manifold.

\subsection{Description of the moves}

The moves that we describe can be broken down into two types, that we call M-moves and $C$-moves. The meaning of the latter is quite clear, as they correspond to so-called elementary collapses and their inverses. The former moves are inspired by the procedure of obtaining a protoMom-structure from an ideal triangulation (see Section 2.1); we describe this origin below in Remark 4.2.

For all the moves considered we introduce a natural notion of admissibility by saying that a move is admissible if it transforms a weak protoMom-structure into a weak protoMom-structure. It will be easy to see from the definition of the moves that this is equivalent to requiring the moves to keep the lateral boundary toral. 
M-moves The precise definition of an M-move is the following one.

Definition 4.1 Let $(M, \Sigma, \Delta)$ be a weak protoMom-structure internal on a compact connected orientable 3 -manifold $N$ with $\partial N=\Sigma$. Let $H$ be a 1 -handle of $\Delta$ of valence at least 1 , and let $\alpha$ be a 2 -handle incident to $H$. Let $\ell \subset \partial M$ be a closed embedded curve such that

(a) $\ell$ is disjoint from the 2 -handles of $\Delta$;

(b) $\ell$ passes exactly 3 times along 1 -handles, counting with multiplicity;

(c) $\ell$ passes along $\partial H$, and the attaching curves of the 2-handles different from $\alpha$ do not separate $\ell$ from the attaching curve of $\alpha$ on $\partial H$;

(d) $\ell$ bounds a disc in the complement of $M$ in $N$.

Then the $M$-move at $(H, \alpha)$ along $\ell$ consists in removing the 2 -handle $\alpha$ and gluing another 2-handle along $\ell$ instead.

The actual shape of an M-move may vary depending on how the curve $\ell$ and the boundary annulus of $\alpha$ pass along the various 1-handles (although the number of possibilities is obviously limited). In Figures 14 and 15 we show two specific examples of an M-move.

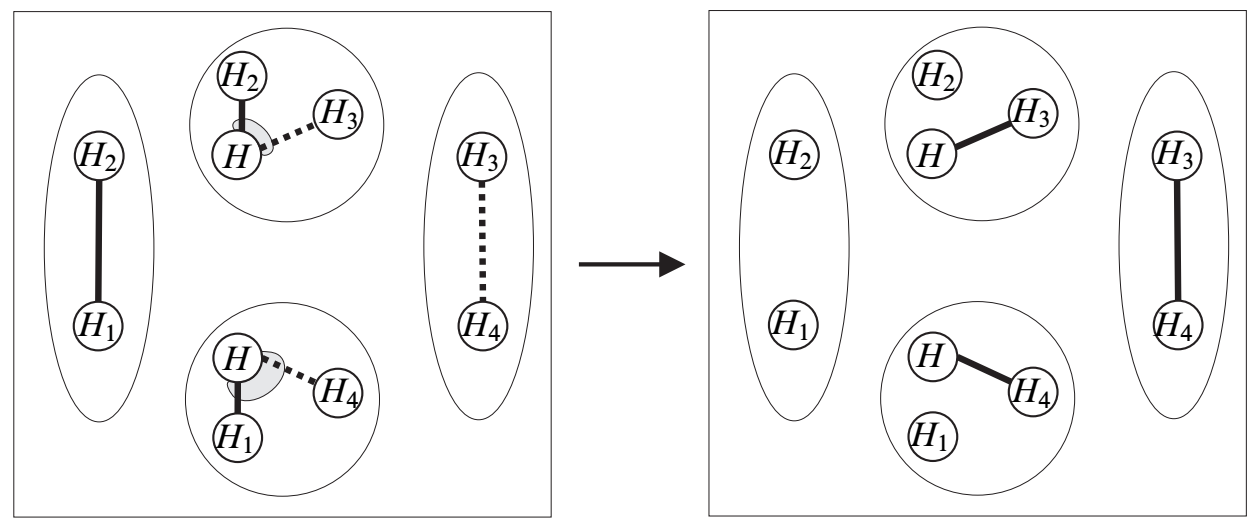

Figure 14. An example of an M-move; on the left, the dotted line indicates segments of the curve $\ell$ and the thick line indicates segments of the core of the boundary annulus of $\alpha$. The areas shown in grey do not intersect any bridge other than that contained in $\alpha$, and the ovals indicate portions of lakes (which actually may or may not belong to the same lake). On the right the thick line indicates segments of the core of the boundary annulus of the 2-handle replacing $\alpha$. 

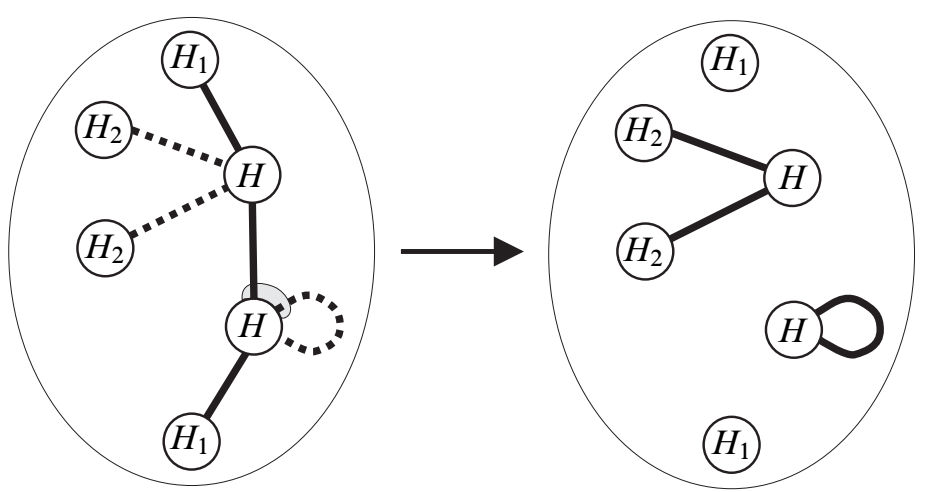

Figure 15. Another example of an M-move; see the explanation for Figure 14. Note that in this case only one lake is involved in the move.

Remark 4.2 The requirements for the curve $\ell$ defining the move may seem rather stringent, so at first glance the question of existence of at least one curve with such properties may appear nontrivial. Note however that the idea of the move comes from considering triangulation-induced protoMom-structures, in particular those obtained by removing some 2-handles from the thickening of the triangulation and keeping all 1-handles; if we have such a protoMom-structure then every tetrahedron such that one of its faces is removed and another one is kept, provides a situation exactly as described in the definition of an M-move.

Remark 4.3 An M-move is not automatically admissible. However, it follows immediately from the definition that it is so if and only if after its application the boundary still consists of tori.

Remark 4.4 It is clear from the definition that the (admissible) M-move performed at $(H, \alpha)$ along $\ell$ is invertible with the (admissible) inverse of the same type. More precisely, the inverse of this move is the M-move performed at $\left(H, \alpha^{\prime}\right)$ along $\ell_{\alpha}$, where $\alpha^{\prime}$ is the 2 -handle inserted by the initial move and $\ell_{\alpha}$ is the gluing curve of $\alpha$ (ie the core circle of its boundary annulus).

C-moves The second class of moves consists of elementary collapses and their inverses, where by an elementary collapse we mean the removal of a 1-handle of valence 1 together with the 2-handle incident to it. The precise description of these moves is as follows.

Definition 4.5 Let $(M, \Sigma, \Delta)$ be a weak protoMom-structure internal on a compact connected orientable 3 -manifold $N$ with $\partial N=\Sigma$, and let $\ell \subset(\partial M \backslash \Sigma)$ be an embedded arc such that 
(a) the endpoints of $\ell$ are contained in the union of the islands;

(b) $\ell$ intersects the union of the 1-handles along precisely two segments and the union of the lakes along precisely one segment;

(c) $\ell$ is disjoint from the 2 -handles of $\Delta$.

Then the $C$-move along $\ell$ consists in first adding a 1 -handle $H$ contained in $N$ and parallel to $\partial M$ and with bases in the immediate vicinity of the endpoints of $\ell$, then completing $\ell$ to a closed curve $\ell^{\prime}$ that passes precisely once along $H$ and is disjoint from any 2 -handles, and finally gluing a new 2 -handle along $\ell^{\prime}$.

The C-move has three distinct shapes that are shown in Figures 16, 17 and 18.

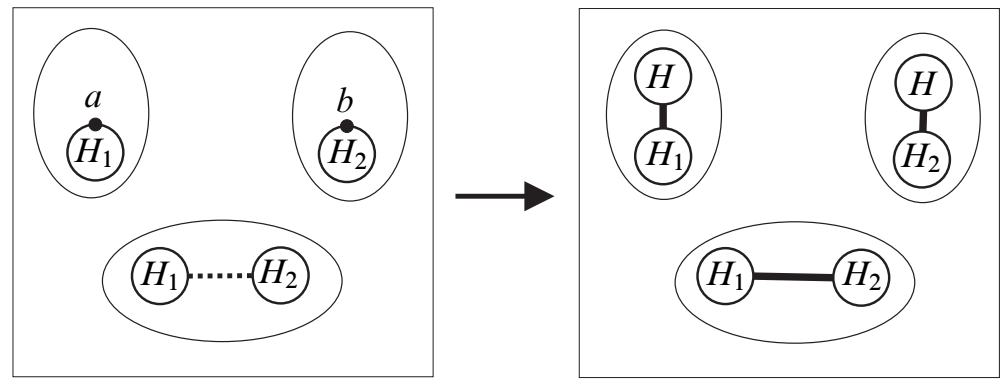

Figure 16. The first type of the C-move; $a$ and $b$ denote the endpoints of $\ell$.

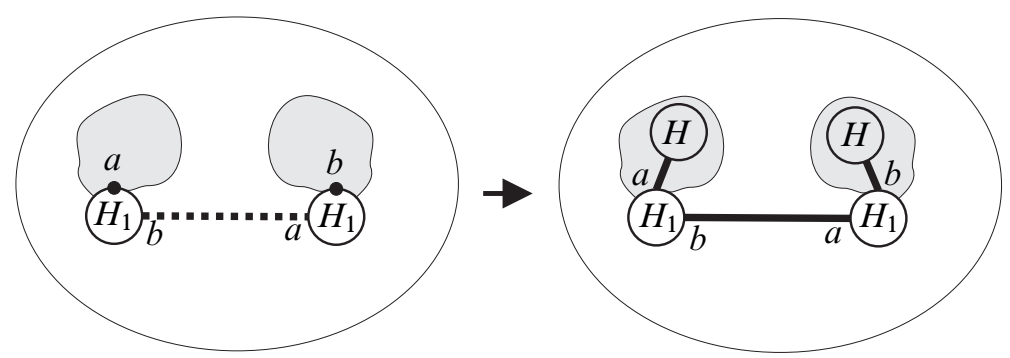

Figure 17. The second type of the $\mathrm{C}$-move; $a$ and $b$ indicate how the segment passes along the 1 -handle $H_{1}$.

It is clear that any C-move is automatically admissible. It is also invertible, with the inverse being a suitable elementary collapse, and we call any of the latter a $\mathrm{C}^{-1}$-move. We will typically refer to all $\mathrm{C}^{ \pm 1}$-moves as simply to C-moves. 


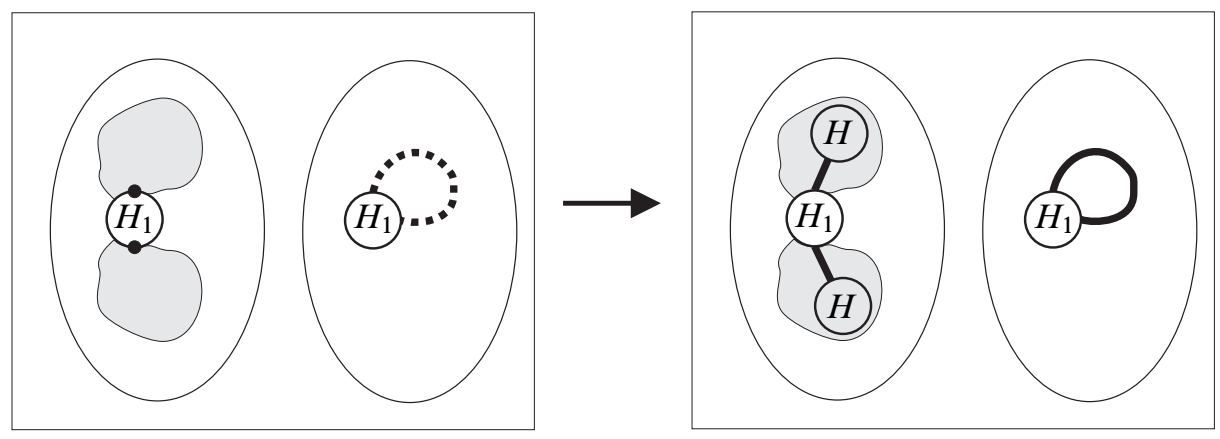

Figure 18. The third type of the C-move

\subsection{Relating protoMom-structures by the moves}

We are now ready to state the main result of the paper.

Theorem 4.6 Let $N$ be a compact connected orientable 3-manifold such that $\partial N$ is a nonempty surface without spherical components. Then any two full weak protoMomstructures internal on $N$ are related by a sequence of admissible $M$ - and $C$-moves.

The proof of this result is based on the statement of Theorem 2.4 and on the construction used to prove it, and on Corollary 3.9. We start by introducing some additional notions and proving some easy facts. For the rest of the section we fix an ambient manifold $N$ as in Theorem 4.6.

General Mom-subgraphs and protoMom-structures Let $\tau$ be an ideal triangulation of $N$; then, as described in Section 1, dual to $\tau$ there is a special spine $P_{\tau}$ of $N$. Note that any general Mom-subgraph $\Gamma$ on $S\left(P_{\tau}\right)$, which is a 4-valent graph, defines a weak protoMom-structure $\left(M_{\Gamma}, \Sigma, \Delta_{\Gamma}\right)$ internal on $N$ : the 1 -handles of $\Delta_{\Gamma}$ are thickenings of the edges of $\tau$, and the 2-handles of $\Delta_{\Gamma}$ are thickenings of the faces of $\tau$ dual to the edges of $S\left(P_{\tau}\right)$ having color $f$ in $\Gamma$. We will call $\left(M_{\Gamma}, \Sigma, \Delta_{\Gamma}\right)$ the protoMom-structure dual to $\Gamma$. This duality extends to the moves:

Proposition 4.7 Let $\tau, \Gamma$, and $\left(M_{\Gamma}, \Sigma, \Delta_{\Gamma}\right)$ be as above, and let $\Gamma^{\prime}$ be obtained from $\Gamma$ by an admissible move of type $(\mathrm{m} 1),(\mathrm{m} 2),\left(\mathrm{m} 2^{\prime}\right),(\mathrm{m} 3)$, or $(\overline{\mathrm{m}} 3)$.

(1) If the move is of type $(\mathrm{m} 1),\left(\mathrm{m} 2^{\prime}\right),(\mathrm{m} 3)$, or $(\overline{\mathrm{m}} 3)$ then the dual structure $\left(M_{\Gamma^{\prime}}, \Sigma, \Delta_{\Gamma^{\prime}}\right)$ is obtained from $\left(M_{\Gamma}, \Sigma, \Delta_{\Gamma}\right)$ by an admissible $M$-move.

(2) If the move is of type (m2) then either $\left(M_{\Gamma^{\prime}}, \Sigma, \Delta_{\Gamma^{\prime}}\right)$ coincides with $\left(M_{\Gamma}, \Sigma, \Delta_{\Gamma}\right)$ or it is obtained from $\left(M_{\Gamma}, \Sigma, \Delta_{\Gamma}\right)$ by an admissible $M$-move. 
Weak protoMom-structures maximal with respect to a triangulation We now present an alternative way to describe the set of protoMom-structures obtained by taking the duals of all Mom-subgraphs in $S\left(P_{\tau}\right)$, where $\tau$ is a fixed ideal triangulation of $N$.

Definition 4.8 We say that a $\tau$-induced weak protoMom-structure $(M, \Sigma, \Delta)$ is $\tau$ maximal if it is not properly contained in any other $\tau$-induced protoMom-structure on $N$.

Remark 4.9 The above definition is equivalent to requiring the set of the 1 -handles of $(M, \Sigma, \Delta)$ to coincide with the set of thickenings of all the edges of $\tau$.

Remark 4.10 When speaking about $\tau$-maximal protoMom-structures, we implicitly fix for each edge or triangle of $\tau$ a specific thickening of it, used then for all $\tau$-induced protoMom-structures.

Lemma 4.11 Let $\tau$ be an ideal triangulation of $N$. Then the set of $\tau$-maximal weak protoMom structures on $N$ is precisely the set of weak protoMom-structures dual to general Mom-subgraphs of $S\left(P_{\tau}\right)$.

Proof A weak protoMom-structure dual to a Mom-subgraph is $\tau$-maximal because it contains all 1-handles, and insertion of some 2-handle would create sphere boundary components. Let now $(M, \Sigma, \Delta)$ be a $\tau$-maximal weak protoMom-structure. Consider the auxiliary coloring $\Gamma^{\prime}$ of $S\left(P_{\tau}\right)$ obtained by assigning color $f$ to the edges dual to the 2-handles that are contained in $\Delta$, and a new color $x$ to all the other edges. Since $\partial M$ has no spherical components, no connected component of $x$-colored edges is contractible; furthermore, $\partial M \backslash \Sigma$ consists of tori, hence we can find a general Mom-subgraph $\Gamma$ such that the set of its $t$-colored edges and $c$-colored edges is contained in the set of $x$-colored edges (relative to $\left.\Gamma^{\prime}\right)$. Then it is clear that $(M, \Sigma, \Delta)$ is contained in $\left(M_{\Gamma}, \Sigma, \Delta_{\Gamma}\right)$, and since $(M, \Sigma, \Delta)$ is $\tau$-maximal, they must actually coincide.

Corollary 3.9 and Proposition 4.7 now imply:

Proposition 4.12 Let $\tau$ be an ideal triangulation of $N$. Then any two $\tau$-maximal weak protoMom-structures are related by a sequence of admissible M-moves.

Recovering a maximal protoMom-structure from an arbitrary one The last essential tool for the proof of Theorem 4.6 is the following: 
Lemma 4.13 Let $(M, \Sigma, \Delta)$ be a full weak protoMom-structure internal on $N$, and let $\tau$ be the triangulation constructed in the proof of Theorem 2.4. Then there exists a sequence of $C$-moves transforming $(M, \Sigma, \Delta)$ into a $\tau$-maximal weak protoMom structure.

Proof Recall from the proof of Theorem 2.4 that $\tau$ can be explicitly constructed by compressing each handle of $\Delta$ on its core and performing a sequence of moves ( $\hat{\mathrm{s}} 1),(\hat{\mathrm{s}} 2)$, ( $(\hat{\mathrm{s}} 3)$ described in Section 2.2 (whose definition would be adjusted in a straightforward manner to account for the fact that we do not compress lakes and the triangles and tetrahedra that we insert are actually truncated). Indeed, recall that the move $\left(\hat{\mathrm{s}} 1^{\prime}\right)$ is actually a composition of ( $(\hat{\mathrm{s}} 1)$ and $(\hat{\mathrm{s}} 2)$ and that the layered triangulation inserted at the conclusion can be obtained by a sequence of ( $\hat{\mathrm{s}} 2)$ moves followed by filling in the one-tetrahedron triangulation of solid torus.

Let us fix such a sequence of ( $\hat{\mathrm{s}} \star)$ moves. To each move we now associate either a $\mathrm{C}$-move or an identity (empty) move on the protoMom-structure $(M, \Sigma, \Delta)$. More precisely, we do the following:

- to each ( $\hat{\mathrm{s}} 1)$ move we associate the C-move that consists in insertion of the 2-handle and the 1-handle that are thickenings of respectively the triangle and the edge inserted by the ( $\hat{\mathrm{s}} 1)$ move under consideration;

- to each ( $\hat{\mathrm{s}} 2)$ move we associate the $\mathrm{C}$-move that consists in insertion of a precisely one 2-handle and one 1-handle where the 1-handle is the thickening of the edge inserted during the ( $\hat{\mathrm{s}} 2)$ move under consideration and the 2-handle is the thickening of precisely one of the two triangles inserted by the move (this association is therefore nonunique);

- no move is associated to any of the ( $(\hat{\mathrm{s}} 3)$ moves.

(Note that we do not associate anything to the final operation of filling in with the one-tetrahedron triangulation). Then the sequence of $(\hat{\mathrm{s}} \star)$ moves fixed above yields a well-defined sequence of C-moves on $(M, \Sigma, \Delta)$. Applying this sequence of moves to $(M, \Sigma, \Delta)$ gives a new weak internal protoMom-structure $\left(M^{\prime}, \Sigma^{\prime}, \Delta^{\prime}\right)$ on $N$. Moreover, $\left(M^{\prime}, \Sigma^{\prime}, \Delta^{\prime}\right)$ is also $\tau$-induced and the set of its 1 -handles coincides with the set of thickenings of all the edges of $\tau$. It now follows from the Euler characteristic argument that $\left(M^{\prime}, \Sigma^{\prime}, \Delta^{\prime}\right)$ is $\tau$-maximal, whence the conclusion.

Proof of Theorem 4.6 It follows from Lemma 4.13 that, given two full weak protoMom-structures internal on $N$, each is related by a sequence of C-moves to a $\tau_{i}-$ maximal weak protoMom-structure for some triangulations $\tau_{i}$ with $i \in\{1,2\}$; Proposition 4.12 then implies that each of the two structures is related by a sequence of 
admissible M- and C-moves to any other $\tau_{i}$-maximal structure, for the corresponding $i$. Recall now [11;13] that two ideal triangulations $\tau_{1}$ and $\tau_{2}$ of $N$ are related to each other by a sequence of instances of the $(2 \rightarrow 3)$-move shown in Figure 19 and of inverses of this move. (This would be false if $\tau_{1}$ or $\tau_{2}$ were to consist of only one

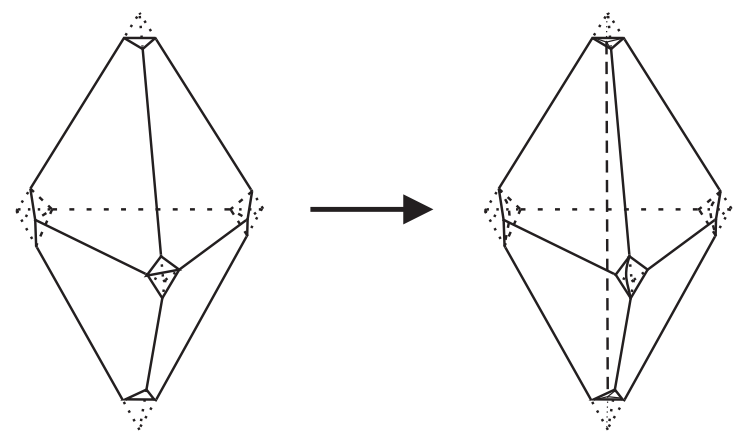

Figure 19. The $(2 \rightarrow 3)-$ move on a truncated triangulation

tetrahedron, but this is impossible for an orientable $N$ without spherical boundary components.)

It is then sufficient to show that if $\tau_{2}$ is obtained from $\tau_{1}$ by one $(2 \rightarrow 3)$-move then there exist a $\tau_{1}$-maximal weak protoMom-structure and a $\tau_{2}$-maximal weak protoMom-structure that are related by M- and C-moves. Indeed, let $\alpha$ be the 2 -handle that thickens the triangle destroyed by the $(2 \rightarrow 3)-$ move, and let $\left(M_{1}^{\prime}, \Sigma, \Delta_{1}^{\prime}\right)$ be any $\tau_{1}$-maximal protoMom-structure not containing $\alpha$; its existence is obvious. Now we note that $\left(M_{1}^{\prime}, \Sigma, \Delta_{1}^{\prime}\right)$ can actually be viewed as a $\tau_{2}$-induced protoMom-structure, and there exists a $\mathrm{C}$-move transforming it into a $\tau_{2}$-maximal structure $\left(M_{2}^{\prime}, \Sigma, \Delta_{2}^{\prime}\right)$; this move consists in inserting the edge and exactly one of the three triangles that appear during the $(2 \rightarrow 3)-$ move and then thickening them. The proof is whence complete.

Remark 4.14 By Theorem 4.6 two genuine (nonweak) Mom-structures internal on the same cusped hyperbolic manifold are related by a sequence of admissible $\mathrm{M}$ - and C-moves. However, even starting with two Mom-structures for which there exists a triangulation with respect to which both structures are maximal, along the sequence one might very well encounter nongenuine (weak in our sense) Mom-structures. Therefore the question of finding combinatorial moves relating to each other any two genuine Mom-structures remains open. 


\section{References}

[1] J DeBlois, P B Shalen, Volume and topology of bounded and closed hyperbolic 3manifolds, Comm. Anal. Geom. 17 (2009) 797-849 MR2643733

[2] D B A Epstein, R C Penner, Euclidean decompositions of noncompact hyperbolic manifolds, J. Differential Geom. 27 (1988) 67-80 MR918457

[3] R Frigerio, B Martelli, C Petronio, Hyperbolic 3-manifolds with non-empty boundary, table (2002) Available at http://www.dm.unipi.it/pages/petronio/ public_html/files/3D/Geo_Bd/geo_bd_\% census.html

[4] D Gabai, R Meyerhoff, P Milley, Minimum volume cusped hyperbolic three-manifolds, J. Amer. Math. Soc. 22 (2009) 1157-1215 MR2525782

[5] D Gabai, R Meyerhoff, P Milley, Mom technology and hyperbolic 3-manifolds, from: "In the tradition of Ahlfors-Bers, V", (M Bonk, J Gilman, H Masur, Y Minsky, M Wolf, editors), Contemp. Math. 510, Amer. Math. Soc., Providence, RI (2010) 84-107 MR2581832

[6] D Gabai, R Meyerhoff, P Milley, Mom technology and volumes of hyperbolic 3_manifolds, Comment. Math. Helv. 86 (2011) 145-188 MR2745279

[7] W Jaco, H Rubinstein, S Tillmann, Minimal triangulations for an infinite family of lens spaces, J. Topol. 2 (2009) 157-180 MR2499441

[8] S Kojima, Polyhedral decomposition of hyperbolic 3-manifolds with totally geodesic boundary, from: "Aspects of low-dimensional manifolds", (Y Matsumoto, S Morita, editors), Adv. Stud. Pure Math. 20, Kinokuniya, Tokyo (1992) 93-112 MR1208308

[9] S Kojima, Polyhedral decomposition of hyperbolic 3-manifolds with totally geodesic boundary, from: "Aspects of low-dimensional manifolds", (Y Matsumoto, S Morita, editors), Adv. Stud. Pure Math. 20, Kinokuniya, Tokyo (1992) 93-112 MR1208308

[10] S Kojima, Y Miyamoto, The smallest hyperbolic 3-manifolds with totally geodesic boundary, J. Differential Geom. 34 (1991) 175-192 MR1114459

[11] S V Matveev, Transformations of special spines, and the Zeeman conjecture, Izv. Akad. Nauk SSSR Ser. Mat. 51 (1987) 1104-1116, 1119 MR925096

[12] P Milley, Minimum volume hyperbolic 3-manifolds, J. Topol. 2 (2009) 181-192 MR2499442

[13] R Piergallini, Standard moves for standard polyhedra and spines, from: "Third National Conference on Topology (Italian) (Trieste, 1986)", Rend. Circ. Mat. Palermo Suppl. 18 (1988) 391-414 MR958750

Dipartimento di Matematica Applicata, University of Pisa

Via F Buonarroti 1C, I-56127 Pisa, Italy

ekaterina.pervova@dma.unipi.it

Received: 17 March $2011 \quad$ Revised: 24 August 2011 